\title{
Parenté après cancer : options actuelles et espoirs pour l'avenir
}

\author{
Conférence multidisciplinaire \\ Houston, 5-7 Mars 2004
} Université du Texas - MD ANDERSON CANCER CENTER

\author{
Eric HUYGHE \\ Groupe de Recherche sur la Fertilité humaine, Toulouse
}

Du 5 au 7 mars 2004, s'est déroulé au M.D. Anderson Cancer Center, Houston, une conférence multidisciplinaire intitulée: "Parenthood after cancer : Today's options and Tomorrow's hopes". Elle était organisée sous le patronage du National Cancer Institute et regroupait les principales équipes de recherches investies sur ce sujet, ainsi que les associations de malades (Lance Armstrong Foundation, Fertile Hope).

\section{Expérience des survivants de cancers concer- nant la fertilité}

Leslie Schover a rappelé que $76 \%$ des survivants de cancers n'ayant pas d'enfants au moment du diagnostic du cancer et $31 \%$ de ceux en ayant déjà, désiraient avoir des enfants dans l'avenir. Quatre vingt dix pour cents des patients déclaraient aussi se sentir en assez bonne santé pour assurer leurs fonctions de père et $80 \%$ pensaient que leur expérience pouvait améliorer leurs qualités de père. À la question "même si je meure jeune, je souhaite être père", 62\% ont répondu oui (Schover).

L'expérience des survivants à montré qu'on ne parlait jamais assez, ni assez tôt, de la fertilité, et a souligné l'importance psychologique de la conservation de sperme.

Aux États-Unis il semble que moins de $25 \%$ des oncologues informent leurs jeunes patients du risque d'infertilité, d'après un rapport de l'organisation Fertile Hope (Nohr).

Une étude émanant du M.D. Anderson Cancer Center, Houston trouvait que moins de la moitié des patients atteints de cancer avaient abordé avec l'oncologue la question de la conception après cancer.

\section{Préservation de la fertilité après cancer}

Chez les hommes atteints de cancers du testicule, la pro- portion d'hommes parvenant à avoir un enfant sans recours à une AMP diminue d'environ 30\% après traitement. La radiothérapie et la chirurgie des masses résiduelles paraissent retentir de manière plus profonde et plus durablement que les chimiothérapies de type BEP (Huyghe, [a]).

Dans tous les cas, l'importance de proposer une cryoconservation de sperme à tous les patients soumis à des thérapeutiques potentiellement stérilisantes est réaffirmée.

La question de la chirurgie conservatrice en cas de tumeur germinale du testicule survenant sur testicule unique ou de tumeur testiculaire bilatérale a également été abordée. Elle permettrait d'éviter les traitements substitutifs androgéniques à vie, mais doit s'accompagner d'une prise en charge systématique du carcinome in situ compte tenu de sa prévalence très élevée (95\%) (Huyghe, [b])

\section{Mécanisme d'atteinte de la fertilité par le cancer et les traitements}

Concernant les mécanismes d'atteinte de la spermatogenèse par les agents chimiothérapiques, les modèles animaux ont permis de mettre en évidence le rôle de $P 53$ et du système Fas comme modulateur de l'activité pro-apoptotique dans le testicule. Le mode d'action du Cisplatine sur la spermatogenèse semble provenir d'une atteinte à la fois des cellules germinales, des cellules de Leydig et de Sertoli. En produisant des ponts-intermoléculaires au sein de l'ADN, non seulement il inhibe la réplication et la trans-

Correspondance :

huyghe.e@chu-toulouse.fr_ 
cription de l'ADN, mais il entraîne également une apoptose. Les spermatocytes sont les principales cellules germinales à mourir par apoptose après exposition au Cisplatine. II y a une élévation à long terme de l'index apoptotique après exposition au Cisplatine, suggérant une altération persistante au niveau de l'épithélium séminifère (Boekelheide).

Concernant la compréhension des mécanismes d'atteinte de la fertilité après radiothérapie, l'équipe de Marvin Meistrich, MDA, Houston, a présenté des résultats très intéressants à partir du modèle animal. Ils ont notamment montré que chez le rat, après exposition à de faible dose de radiation, on n'observait pas de destruction des spermatogonies, mais plutôt un blocage de la spermatogenèse par impossibilité des spermatogonies à se différencier. Ils ont également mis en évidence que la spermatogenèse et la fertilité pouvaient être restaurées chez le rat en diminuant le taux de testostérone intra-testiculaire avec un analogue ou un antagoniste de la GnRH (Shetty et Meistrich).

La même équipe a également montré qu'il existait un lien entre la restauration de la spermatogenèse chez le rat, le taux de testostérone intra testiculaire, et des phénomènes d'oedème intra-testiculaire (Porter et Meistrich).

Cependant ces résultats n'ont pas été retrouvés chez le singe.

L'équipe d'Alexander Giwercman, Malmö, Suède, a présenté les résultats d'une étude portant sur l'impact des thérapies, le polymorphisme des récepteurs aux androgènes, et la qualité du sperme en cas de cancer de testicule. Ils ont révélé qu'il existait une corrélation inversement proportionnelle entre la sensibilité aux androgènes, et la longueur des séquences répétitives CAG du récepteur aux androgènes et la restauration de la spermatogenèse après chimiothérapie BEP 3 ou 4 cycles (Eberhard et Giwercman).

Cette même équipe a également montré qu'après radiothérapie, l'index de fragmentation de l'ADN était augmenté, et non après chimiothérapie BEP (Stahl).

\section{Santé des enfants de patients ayant eu un cancer}

Une étude récente menée au sein du département de pharmacologie et de thérapeutique de l'université Mc Gill de Montréal, Canada, a étudié les effets du cyclophosphamide sur le conceptus à partir d'un modèle animal (rat). Elle a montré que de faibles doses au long cours chez le mâle augmentaient le taux de malformations. Les effets étaient influencés par le stade des cellules germinales lors de la première exposition à la drogue. Les traitements au long cours diminuaient de manière nette l'expression des gènes de réponse au stress dans les spermatocytes pachytènes et les spermatides rondes, mais non dans les spermatides allongées.

La diminution de l'expression des gênes de réponse au stress dans les cellules germinales pourrait permettre aux altérations de l'ADN de s'accumuler. Une augmentation dose dépendante des altérations de l'ADN a été observée 3 semaines après exposition à la drogue. L'exposition pen- dant 9 semaines augmentait la fréquence des disomies et des nullisomies. Après fécondation, les spermatozoïdes des mâles traités par le cyclophosphamide se décondensaient plus rapidement que les spermatozoïdes du groupe contrôle et la formation du pronucléus était plus précoce. La synthèse d'ARN était plus élevée chez les embryons à 1 cellule provenant de mâles traités, par rapport aux témoins. L'expression des gènes spécifiques était altérée chez les embryons provenant des mâles traités dès le stade 1 cellule. Donc l'exposition paternelle temporaire dérégule l'activation des gènes chez le zygote, altérant l'horloge du développement (Hales).

Concernant la santé des enfants de patients ayant eu un cancer, Sophie Fossa a présenté les résultats d'une étude réalisée à partir du registre du cancer de Norvège et des cas recensés au Norvergian Radium Hospital d'Oslo, Norvège. 2307 enfant sont nés de 13817 patients. Comparés à la population norvégienne, ces enfants avaient un poids de naissance plus bas et étaient plus souvent prématurés, mais aucune augmentation des malformations n'a été objectivée, et on ne recensait pas d'augmentation des complications obstétricales ou néonatales (Fossa).

\section{Considérations socio-économiques et culturelles}

Contrairement à ce que nous connaissons en France, aux États-Unis, les conditions socio-économiques influent sur le choix des patients quant à leur fertilité. En effet les coûts des techniques d'assistance médicale à la procréation (AMP) et de mise en banque de sperme et tissus sont infiniment supérieurs à ce qu'ils sont en France (une année de conservation de sperme revient environ à 500 dollars, et une conservation d'embryons à 10000 dollars).

Par ailleurs, une étude menée au Texas a montré qu'il existait un problème d'accès aux soins de certains groupes ethniques pour des raisons éducationnelles, culturelles, et socio-économiques (Jenkins).

C'est le cas de la communauté afro-américaine qui a volontiers tendance à se tourner vers l'adoption, plutôt que vers une AMP en cas de stérilité. Pour les communautés mexicaine et noire américaine, le poids de la religion semble également un frein à l'AMP et même à la conservation de sperme, qui sont parfois interprétées comme contraires à la volonté de Dieu.

\section{Voies d'avenir pour la recherche et les pra- tiques cliniques}

Les progrès visent surtout à permettre de préserver la fertilité des patients pré-pubères atteints de cancers. II est estimé que 1 enfant sur 650 se verra diagnostiquer un cancer avant l'âge de seize ans et que celui-ci sera guéri dans $80 \%$ des cas. Environ $20 \%$ des garçons seront stériles après traitement. Concernant les voies d'avenir pour préserver un potentiel de fertilité chez les patients atteints de cancer, trois stratégies sont à l'étude (Patrizio; Schlatt).

a) La première est de réaliser une biopsie testiculaire, de la 
cryoconserver puis de la transplanter après traitement. Le problème de cette technique est le risque de récidive du cancer.

b) La seconde technique est la transplantation de spermatogonies autologues. Cependant, il est très difficile d'isoler les cellules souches parce qu'elles n'ont pas de marqueurs morphologiques, antigéniques ou biochimiques. Ces techniques posent le problème de la contamination des préparations de tissu testiculaire par des cellules tumorales. La technique de transplantation de spermatogonies est en voie d'évaluation chez la souris. Les premières expériences ont montré qu'on pouvait obtenir des spermatogonies humaines après transplantation de cellules souches dans les tubes séminifères de souris immunodéficiantes. Cependant, si les spermatogonies adultes ont pu coloniser les tubes séminifères, et survivre pendant plus de six mois, elles n'ont pas donné lieu à une spermatogenèse.

Le transfert de cellules germinales chez le singe est en voie d'évaluation.

c) Une troisième stratégie est représentée par les xénogreffes ectopiques de tissu testiculaire. De petits morceaux de tissu testiculaire de nouveau-nés provenant de différentes espèces (souris, cochons, singe) ont été greffés dans le tissu sous cutané de souris immunodéficiantes castrées, permettant l'induction d'une spermatogenèse. Une étude récente a fait état de xénogreffe de tissu testiculaire adulte provenant d'hommes présentant une azoospermie obstructive et non obstructive chez la souris avec analyse à 15 jours, 1 et 2 mois. Les xénogreffes ont survécu et ont établi une vascularisation, mais une dégénérescence des tubules séminifères est survenue.

Compte tenu des progrès rapides dans le développement de nouvelles stratégies pour générer des spermatozoïdes à partir de cellules germinales spermatogoniales ou de tissu testiculaire immature, la recommandation a été faite de conserver chaque fois que possible du tissu testiculaire en cas de cancer chez l'enfant pré-pubère. On estime que dans un délai de 10 à 15 ans, il sera possible de produire des spermatozoïdes à partir de cellules souches cryoconservées.

Cependant, avant une application clinique, des problèmes éthiques et techniques subsistent.

\section{RÉFÉRENCES}

Boekelheide, K. : Mechanisms of toxic damage to spermatogenesis. (communication)

Eberhard, J. et al. : Impact of therapy and androgen receptor polymorphism on semen quality in men treated for testicular germ cell cancer. (poster)

Fossa, S. D. : Parenthood in survivors after adulthood cancer and perinatal health in their offspring. (communication)

Hales, B. F. et al. : Impact of paternal exposure to chemotherapy on offspring. (communication)

Huyghe, E. et al. (a): Fertility after cancer treatment: results of a large multicenter study. (poster)

Huyghe, E. et al. (b) : What can be preserved, by performing a conservative surgery for germ cell testicular tumors? (poster)

Jenkins, R. L. : Ensuring access to education and services on infertility for the underserved. (communication)

Nohr, L. : Options for parenthood after cancer. (communication)

Patrizio, P. et al. : Experience with xenografts and issues for safe usage in humans. (communication)

Porter, K. L. : Effect of testicular edema on spermatogenesis in irradiated rat testes. (poster)

Schlatt, S. Cryopreservation and transplantation of spermatogonia for preservation of male fertility. (communication)

Schover, L. R. : The cancer experience and motivation for biologi$\mathrm{cal}$ and social parenthood. (communication)

Shetty, G. : Hormonal approaches to preservation and restauration of male fertility after cancer. (communication)

Stahl, $O$. et al. : The impact of testicular cancer and its treatment on sperm DNA integrity. (poster) 